\title{
Cell therapy for acute myocardial infarction: where are we heading?
}

\author{
Kai C Wollert and Helmut Drexler
}

Repair of the heart is an old dream of physicians caring for patients with myocardial infarction. Despite mechanical reperfusion and advances in pharmacotherapy, however, the repair of infarcted myocardium mainly by endogenous repair has not been achieved, and healing remains as being mainly by scar formation. In this context, the emerging concept of adult stem-cell plasticity has generated great excitement among molecular and clinical cardiologists.

Stem cells are undifferentiated cells capable of self-replication, transformation into dedicated progenitor cells, and differentiation into specialized progeny. Bone marrow is a rich source of various stem-cell and progenitor-cell populations, including, hematopoietic stem and progenitor cells, mesenchymal stem cells, and endothelial progenitor cells. The concept of stem-cell plasticity suggests that stem cells can transdifferentiate into distinct mature phenotypes when placed in a tissue-specific microenvironment. Remarkably, acutely infarcted myocardium facilitates mobilization of stem and progenitor cells from the bone marrow. Moreover, neoangiogenesis and myogenesis have been reported after transplantation of stem and progenitor cells in animal models of myocardial infarction, usually associated with improved contractile function.

These observations have created great enthusiasm in some cardiologists but skepticism in others. Yet, shortly after these experimental observations were made, several small-scale clinical trials of cell therapy were initiated. Because of the ease of harvest and no need for ex vivo manipulation, most investigators have used unselected autologous bone marrow cells. In the meantime, one randomized clinical trial and several observational trials indicate that intracoronary infusion of bone-marrow cells enhances left-ventricular function after myocardial infarction.

Ironically, while cell therapy is being introduced into the clinical setting, new experimental data bring into question whether myogenesis contributes to the beneficial effects of cell therapy. The controversy revolves
Should the slow

progress in

understanding

the basic

science of how

cell therapy

works affect

how quickly we

proceed with

clinical trials?

KC Wollert is an

Assistant Professor and

$H$ Drexler is the Chief

of the Department

of Cardiology and

Angiology, Hannover

Medical School,

Hannover, Germany.

$H$ Drexler is also

an advisory board

member for Nature

Clinical Practice

Cardiovascular

Medicine.

Competing interests

The authors declared

they have no competing

interests.

www.nature.com/clinicalpractice doi:10.1038/ncpcardio0053 around whether hematopoietic stem cells transdiffentiate into cardiomyocytes when injected into nonreperfused, infarcted mouse myocardium. Some commentators advocate that further clinical investigation be halted until it is resolved. Yet, although data have been presented to support and to refute that hypothesis, both sides probably agree that hematopoietic stemcell transplantation improves mouse cardiac function after infarction.

Clearly, the mechanisms of how bone-marrowcell transfer improves cardiac function in patients with reperfused infarcts remain enigmatic. Beyond the possibility of transdifferentiation, studies have highlighted the potential of bonemarrow cells to promote paracrine effects in ischemic tissues, indicating that paracrine signaling rather than cell incorporation might promote functional recovery.

Should the slow progress in understanding the basic science of how cell therapy works affect how quickly we proceed with clinical trials? Let us assume that the clinical approach is safe and efficacious in patients. Why should we wait until basic scientists have worked out the underlying mechanism? After all, numerous drugs have been used efficaciously without a complete understanding of their mechanisms of action. Obviously, safety is the key issue.

So far, published experience suggests that bone-marrow-cell transfer in patients with myocardial infarction is safe short-term and midterm with no major adverse effects or procedurerelated deaths. The field now is rapidly moving towards intermediate-size, double-blind trials to gather longer-term follow-up data. Ultimately, a large outcome trial will have to be conducted. We need to proceed cautiously with carefully designed, clinical trials. At the same time, continued basic research to elucidate the underlying mechanism of cell therapy is clearly needed.

Further discussion of many of the issues related to cell therapy is presented in two papers by Noel Caplice et al. in this and next month's issues. 